\title{
Oscillatory Persistent Currents in Self-Assembled Quantum Rings
}

\author{
N. A. J. M. Kleemans, ${ }^{1}$ I. M. A. Bominaar-Silkens, ${ }^{2}$ V. M. Fomin,,${ }^{1,3,4}$ V. N. Gladilin,,${ }^{3,4}$ D. Granados, ${ }^{5}$ A. G. Taboada, ${ }^{5}$ \\ J. M. García, ${ }^{5}$ P. Offermans, ${ }^{1}$ U. Zeitler, ${ }^{2}$ P. C. M. Christianen, ${ }^{2}$ J. C. Maan, ${ }^{2}$ J. T. Devreese, ${ }^{1,3}$ and P. M. Koenraad ${ }^{1}$ \\ ${ }^{1}$ PSN, COBRA, Eindhoven University of Technology, The Netherlands \\ ${ }^{2}$ HFML, IMM, Radboud University Nijmegen, The Netherlands \\ ${ }^{3}$ TFVS, Universiteit Antwerpen, Belgium \\ ${ }^{4}$ FSM, Universitatea de Stat din Moldova, Chisinau, Moldova \\ ${ }^{5}$ CSIC, Instituto de Microelectrónica de Madrid, Spain
}

(Received 14 March 2007; published 5 October 2007)

\begin{abstract}
We report the direct measurement of the persistent current carried by a single electron by means of magnetization experiments on self-assembled InAs/GaAs quantum rings. We measured the first Aharonov-Bohm oscillation at a field of $14 \mathrm{~T}$, in perfect agreement with our model based on the structural properties determined by cross-sectional scanning tunneling microscopy measurements. The observed oscillation magnitude of the magnetic moment per electron is remarkably large for the topology of our nanostructures, which are singly connected and exhibit a pronounced shape asymmetry.
\end{abstract}

PACS numbers: 73.21.La, 73.23.Ra, 78.67.Hc

In quantum mechanics, particular attention is paid to phenomena occurring due to the phase coherence of charge carriers in doubly connected (ring) topologies. Electrons confined to a submicron ring manifest a topologically determined quantum-interference phenomenon, known as the Aharonov-Bohm (AB) effect [1], as a result of the oscillatory behavior of their energy levels as a function of an applied magnetic field. This behavior is usually associated with the occurrence of oscillatory persistent currents in the ring [2-4]. Experimental evidence for $A B$ oscillations has been detected in the mesoscopic regime in metallic $[5,6]$ and semiconducting $[7,8]$ rings, containing many electrons. We address the occurrence of the $\mathrm{AB}$ effect in defect-free self-assembled semiconductor nanostructures [9-13]. The ability to fill nanostructures with only a few (1-2) electrons offers the unique possibility to detect magnetic field induced oscillations in the persistent current carried by single electron states. We report the first direct measurement by means of ultrasensitive magnetization experiments of the oscillatory persistent current carried by a single electron in self-assembled InAs/GaAs "volcanolike" nanostructures. Remarkably, this single electron current occurs even in the absence of an opening [14] in our nanostructures, which is required for the $A B$ effect in the standard treatment [1]. The magnetic field at which the first oscillation in the magnetic moment arises is much higher than expected from the diameter of the quantum rings as determined by atomic force microscopy [13]. However, the experiments are in good agreement with a model based on the structural parameters as determined with cross-sectional scanning tunneling microscopy (XSTM) measurements.

The persistent current was determined via the magnetic moment of electrons in a highly homogeneous ensemble of InAs self-assembled nanostructures. The sample was grown by molecular beam epitaxy and contains 29 mutually decoupled periods [Fig. 1(a)] [15]. Each period con- sists of a nanostructured InAs layer, between two $24 \mathrm{~nm}$ GaAs layers, and a $2 \mathrm{~nm}$ doped $\left(7 \times 10^{16} \mathrm{~cm}^{-3} \mathrm{Si}\right) \mathrm{GaAs}$ layer that provides electrons to the InAs nanostructures. We used a one-dimensional Poisson solver [16] to estimate the average number of electrons per nanostructure to be about 1.5. Considering the two possible spin orientations we assume that all electrons occupy the orbital ground state. The sample is capped by a final nanostructured layer. By performing atomic force microscopy (AFM) on this layer, the nanostructure density was determined to be $9 \times$ $10^{9} \mathrm{~cm}^{-2}$ per layer. Photoluminescence (PL) experiments showed a single peak, indicating a unimodal size distribution, at $1.3 \mathrm{eV}$, which is typical for these nanostructures [10]. The FWHM of the PL peak is $40 \mathrm{meV}$, from which we estimated a size dispersion of about 5\%. It was shown [14] that performing AFM on the top nanostructured layer is not suitable to determine the actual dimensions of a nanostucture. Therefore a reference sample was grown under nominally identical growth conditions as the sample mentioned before, but now on a conductive substrate, suitable for XSTM characterization. The XSTM images revealed structures of about $11.5 \mathrm{~nm}$ radius [Fig. 1(b)] [14]. Most importantly, the shape of these nanostructures differs considerably from that of ideal rings in two respects: (i) the presence of indium in their center resulting in the absence of a hole in the nanostructures and (ii) a distinct anisotropy of the nanostructure, i.e., the height of the rim is larger in the [110] than in the [110] direction $[10,17]$.

The magnetic moment of the nanostructures is obtained from magnetization experiments using a torque magnetometer [18]. These measurements were performed at temperatures of $T=1.2 \mathrm{~K}$ and $T=4.2 \mathrm{~K}$ in magnetic fields up to $15 \mathrm{~T}$. The total magnetization of the sample is due to about $1.5 \times 10^{11}$ nanostructures with a total number of electrons $N \sim 2.2 \times 10^{11}$. These numbers are based on the AFM measurements, the sample size of $7 \times 8 \mathrm{~mm}^{2}$, the Si flux during the growth, the thickness, and the number 


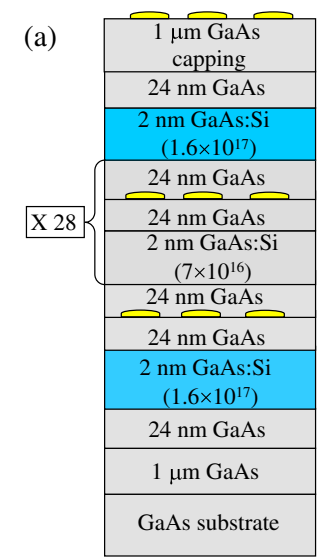

(c)
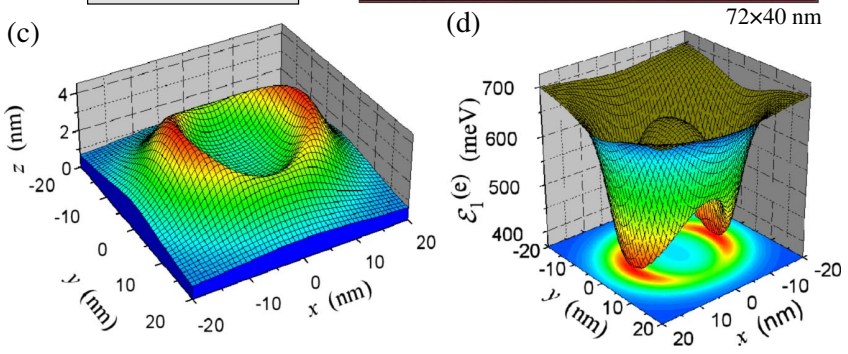

FIG. 1 (color online). (a) Layer structure of the InAs/GaAs sample for magnetization, consisting of 29 layers of selfassembled InAs nanostructures. The nanostructures are located between two $24 \mathrm{~nm}$ GaAs layers. The repeated sequence contains a modulation doping layer, which provides electrons to the nanostructured layers. Additional doping layers (blue or dark gray) are inserted to accommodate for the depletion toward the capping layer and the undoped substrate. (b) XSTM images of different cross-sections of two stacked nanovolcanoes in the reference sample. The images reveal the presence of indium (bright spots) in the center of the nanostructures, and a clear difference in the height of the rim between the [110] and [110] directions. This characterization leads to a realistic description of (c) the height profile and (d) the adiabatic potential for an electron in the nanostucture.

of the doping layers. Figure 2(a) shows the raw experimental data as a function of the magnetic field $B$ for $T=$ $4.2 \mathrm{~K}$ and $T=1.2 \mathrm{~K}$ [19]. Over the entire magnetic field range we observed a relatively large background signal, which is due to the substrate and to dia- and paramagnetic materials close to the sample [20-22]. On top of this background, one clearly observes an oscillation of the magnetization at a magnetic field of about $14 \mathrm{~T}$. To enhance the visibility of this oscillation, a linear background is subtracted from the signal. Furthermore, we normalized the signal to the total number of electrons $N$ in the sample, resulting in the magnetic moment per electron $\mu=M / N$ [Fig. 2(b)]. The sensitivity of the magnetometer is $2.8 \times$ $10^{-12} \mathrm{~J} / \mathrm{T}$, i.e., $3 \times 10^{11} \mu_{B}$ at $B=14 \mathrm{~T}$, and it is limited by mechanical noise, which is about $8 \%$ of the experimentally observed oscillation magnitude.

To prove that the observed oscillation is not an artifact due to the background subtraction, we also plot the first derivative of the signal [inset to Fig. 2(b)], which is much
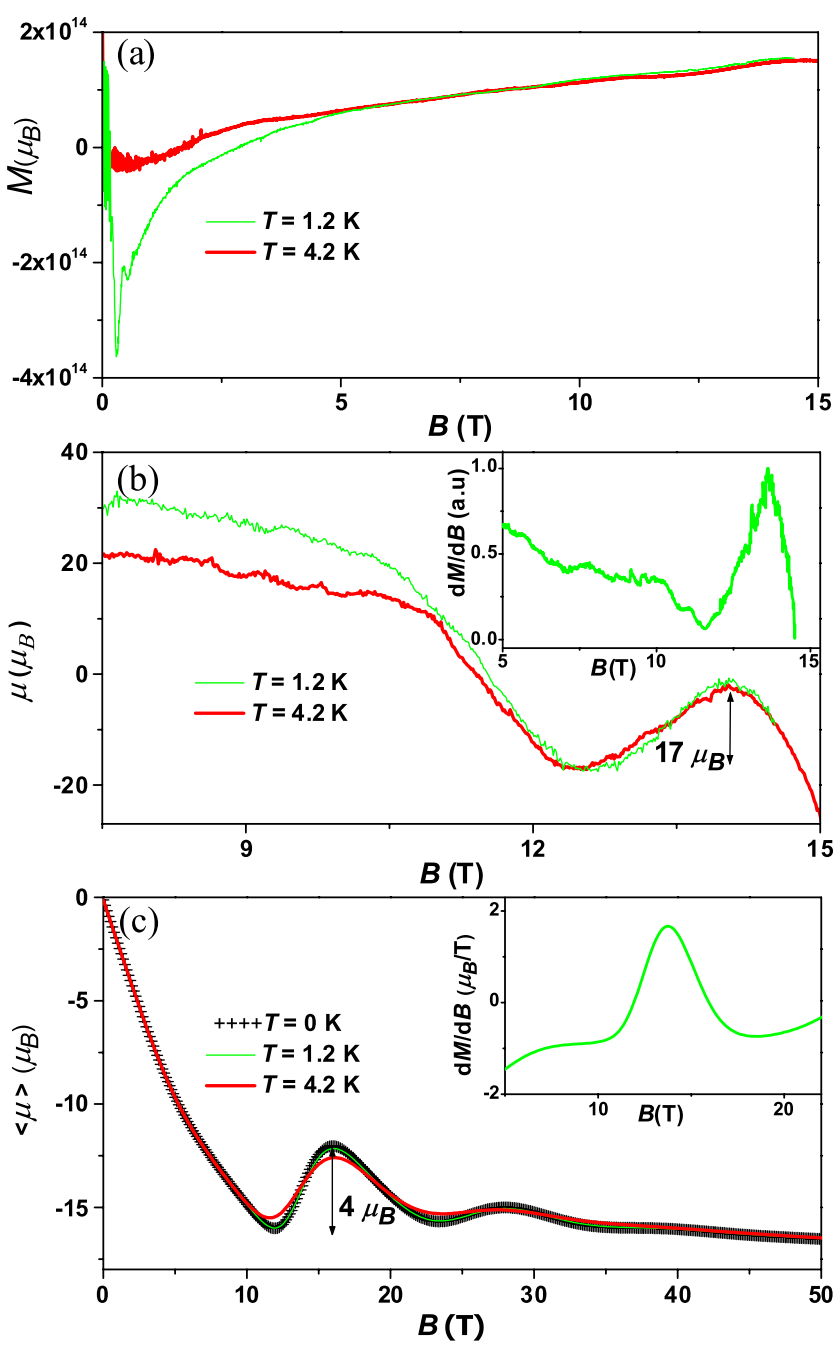

FIG. 2 (color online). (a) Experimental curve of the magnetic moment $M$ at $T=4.2 \mathrm{~K}$ and $1.2 \mathrm{~K}$. An Aharonov-Bohm oscillation is observed around $14 \mathrm{~T}$, superimposed on a smooth background. (b) Oscillation in the magnetic moment per electron $(\mu=M / N)$, obtained at $1.2 \mathrm{~K}$ and at $4.2 \mathrm{~K}$, after subtracting the linear background from the signal, dividing by the total number of electrons $N$, and averaging over several measurements. The inset shows the first derivative of the experimental magnetic moment with respect to $B$ at $T=1.2 \mathrm{~K}$. (c) Calculated magnetic moment, and its derivative (inset), of a single electron in a nanostructure at different temperatures. The calculations are based on the structural parameters of the nanovolcanoes, as obtained by XSTM measurements, and accurately reproduce the Aharonov-Bohm oscillation at $B=14 \mathrm{~T}$.

less sensitive to the monotonous background. This procedure reveals an oscillation around $14 \mathrm{~T}$ as a fingerprint of the $A B$ effect. We performed several tests to ensure that the oscillatory signal does originate from the magnetic moments of the ensemble of nanostructures. First, we polished away the epilayers of the sample, leaving behind only the substrate, and repeated the magnetization experiment. This measurement yielded a smooth background magnetization curve without an oscillation around 14 T. Second, we performed Shubnikov-de Haas experiments by measuring 
the magnetoresistance of the sample up to magnetic fields of $21 \mathrm{~T}$. We found a high value of resistivity without any oscillatory behavior, which proves that there are no free carriers in the epilayers. Finally, we verified that the oscillatory signal is not related to de Haas-van Alphen oscillations [23]. By estimating the electron density that would be necessary to reproduce the measured oscillation period, we found an electron density, which is 2 orders of magnitude larger than is expected from the doping levels. Therefore we conclude that the observed oscillations in the magnetization are caused by the electrons confined in the self-assembled nanostructures.

A first step to interpret this result is to compare our quantum ring to an ideal $\mathrm{In}_{0.55} \mathrm{Ga}_{0.45}$ As ring with a similar diameter of $11.5 \mathrm{~nm}$ [14]. The electron energy $E^{(e)}$ spectrum as a function of the magnetic field, for such a ring, is shown in Fig. 3(a). The arrow indicates a changeover from the state with angular momentum $\ell=0$ to the state with $\ell=-1$ as the ground state. The magnetic moment for a single electron by $\mu=-\frac{\partial F}{\partial B}$ is calculated from $F$, the free energy of the system. The changeover of the ground state of an ideal ring induces a jump in the magnetization around $5 \mathrm{~T}$. As we observe a distinct oscillation near $14 \mathrm{~T}$, the simple model of an ideal ring is clearly not sufficient to describe our volcanolike nanostructures. Therefore we determine the energy spectrum as a function of the magnetic field of a more realistic model based on the XSTM characterization of the nanostructure [14] [cf. the scheme in Fig. 1(c)].

Figure 1(d) shows the in-plane adiabatic potential $\mathcal{E}_{1}^{(e)}(x, y)$ for a single electron in a volcanolike nanostruc-
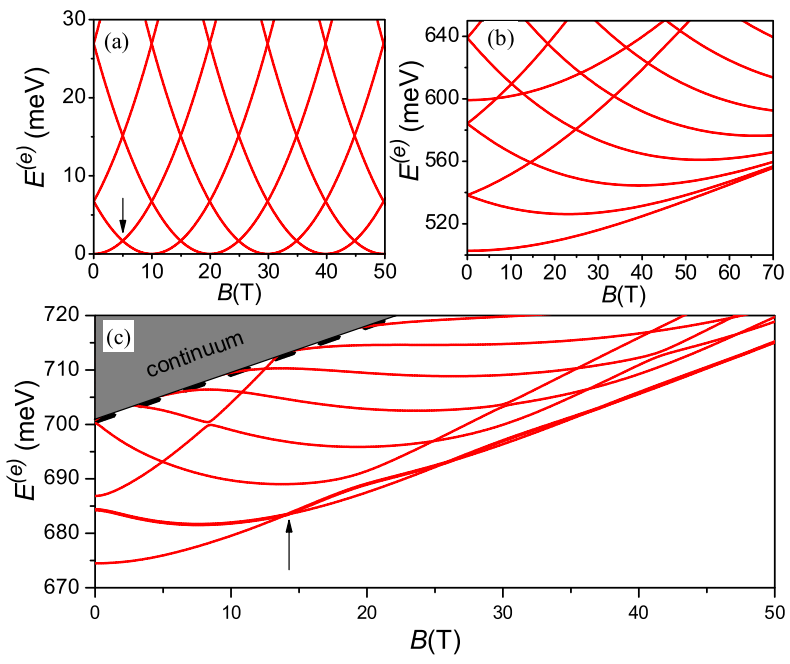

FIG. 3 (color online). (a) The electron energy levels $E^{(e)}$ as a function of magnetic field for an ideal ring with radius $11.5 \mathrm{~nm}$. The arrow indicates a changeover from a state with $\ell=0$ to a state with $\ell=-1$ as the ground state, which takes place around 5 T. (b) $E^{(e)}$ as a function of $B$ for a disc-shaped quantum dot. There are no crossings of the lower lying energy levels. (c) The realistic model of the volcanolike nanostructure reveals a changeover around $14 \mathrm{~T}$. ture. The potential has a finite central maximum (at $x=$ $y=0$ ), which makes the structure singly connected, as distinct from a doubly connected ideal ring. Furthermore, the potential possesses two pronounced minima (at $x=$ $\pm 11.5 \mathrm{~nm}, y=0$ ) separated by potential barriers (at $x=$ $0, y= \pm 11.5 \mathrm{~nm}$ ), reflecting the anisotropy of the nanostructure. We used a model based on the Hamiltonian of an electron in a strained self-assembled nanostructure [24,25] and included piezo-electric effects [26]. The strain tensor and the distribution of indium for the volcanolike geometry of the self-assembled nanostructures were calculated following a three-dimensional finite-element method of elasticity theory [27]. The Schrödinger equation was solved using the adiabatic approximation, separating electron degrees of freedom into "fast" (the motion along the growth axis) and "slow" (the in-plane motion) components. With the anisotropic adiabatic potential $\mathcal{E}_{1}^{(e)}$ as depicted in Fig. 1(d), the Schrödinger equation for the slow degrees of freedom determines the eigenstates of the in-plane motion. Finally, the electron energy eigenvalues in the nanostructure were obtained by diagonalizing the adiabatic Hamiltonian for the slow degrees of freedom in the basis of the in-plane wave functions with 20 radial and 25 azimuthal functions [19]. The resulting electron energy spectrum is shown in Fig. 3(c). The corresponding magnetization curve is plotted in Fig. 2(c), where we averaged over a nanostructure ensemble with a size dispersion of 5\%, consistent with the width of the PL peak.

Most importantly, our model accurately explains the position of the observed $\mathrm{AB}$ oscillation around $14 \mathrm{~T}$, rather than at $5 \mathrm{~T}$ expected for an ideal ring of the same radius. The difference in the position of the $\mathrm{AB}$ oscillations is due to the influence of strain in the self-assembled volcanolike nanostructures as well as to the singly connectedness of these nanostructures. At low fields the electronic ground state has a dominant component with angular momentum zero and is relatively sensitive to the confining potential. In an ideal ring of radius $R$ the ground state wave function is concentrated at $\rho=R$ and vanishes at the center of the ring $(\rho=0)$, where the potential is infinitely high. For a singly connected structure the adiabatic potential has a finite height and thus a nonzero wave function at $\rho=0$. In comparison to an ideal ring, the electron density is therefore shifted toward the center, leading to a smaller effective radius and a higher magnetic field for the first $\mathrm{AB}$ oscillation. We also compare our volcanolike nanostructure with a disc-shaped quantum dot. Although both nanostructures are singly connected, the behavior of the discshaped quantum dot is fundamentally different: no energy crossings occur for the lower energy levels [compare Figs. 3(b) and 3(c)] and consequently there are no AB oscillations due to the absence of a central maximum of the potential. Therefore, the actual profile of the confining potential is essential to interpret the phase-coherent electronic properties of these nanostructures.

Figure 2(c) also shows the calculated magnetic moment for higher magnetic fields that are not yet accessible by 
magnetization experiments. Comparison of the higher order $\mathrm{AB}$ oscillations in realistic nanostructures with those in ideal quantum rings discloses two major differences. First, the ratio of magnetic fields, at which the two first jumps in the magnetic moment occur, is equal to $1: 3$ for ideal rings [Fig. 3(a)] and is approximately 1:2 for the self-assembled volcanolike nanostructures. As stated above, a diminished effective radius of the electron state with $\ell=0$ in the nanostructure leads to a pronounced shift of the first $A B$ oscillation toward higher fields. This effect is less prominent for the states with higher angular momentum $\ell$ that determine the ground state for higher fields. As a result the shift of the $\mathrm{AB}$ oscillations toward higher fields is relatively smaller for states with higher $\ell$. The second difference between the calculated results for the volcanolike nanostructures and for the ideal rings consists in that for realistic nanostructures the higher order $\mathrm{AB}$ oscillations are strongly damped. This is a consequence of the presence of the magnetic field in the rim of the nanostructures, which enhances the electron localization close to the minima of the adiabatic potential.

In Fig. 2(c) the calculated results are plotted for three different temperatures. Without including size variations of the nanostructures, the calculated amplitude of the $\mathrm{AB}$ oscillations increases with decreasing temperature. However, a negligible temperature effect on the electron magnetic moment in our model [see Fig. 2(c)] is due to the nanostructure ensemble averaging, as was observed experimentally [see Fig. 2(b)]. As a final remark, the experimental value for the oscillation magnitude of the magnetic moment per electron, $\Delta \mu \sim 17 \mu_{B}$, is higher than the $4 \mu_{B}$, which we calculated based on the XSTM analysis. Calculations showed that the magnitude is sensitive to the exact structural properties, such as the indium concentration and the shape. A magnitude of $17 \mu_{B}$ at the observed transition magnetic field position is readily achieved for slightly modified nanostructures. It is realistic to assume that the nanostructures in the sample used for the magnetization measurements are somewhat different from those in the reference sample used for the XSTM characterization. Furthermore, the number of electrons confined in the quantum rings and the size and shape of the quantum rings determine the magnetization as a function of the magnetic field [28]. It is therefore interesting to vary the doping concentration in order to detect the effect of the number of electrons confined in the quantum ring on the magnetization behavior.

In conclusion, we demonstrate, using advanced growth capabilities, experimental characterization and theoretical modeling, the existence of an oscillatory persistent current in self-assembled nanostructures containing only a single electron. Even though the nanostructures under investigation are singly connected and anisotropic, they show the $\mathrm{AB}$ behavior that is generally considered to be restricted to ideal (doubly connected) topologies. These results demon- strate the possibility to design and fabricate nonmagnetic semiconductors with magnetic properties, which can be controlled by tuning the size and shape of self-assembled nanostructures.

This work is part of the research program of NanoNed and FOM, which are financially supported by the NWO (The Netherlands). This work was supported by the FWO$\mathrm{V}$ projects No. G.0435.03, No. G.0449.04, the WOG No. WO.035.04N (Belgium), the NANOSELF II (No. TEC-2005-05781-C03-01), the MEC (ConsoliderIngenio 2010 "QOIT", No. CSD2006-00019) (Spain) and the EC SANDiE Network of Excellence (No. NMP4CT-2004-500101).

[1] Y. Aharonov and D. Bohm, Phys. Rev. 115, 485 (1959).

[2] N. Byers and C. Yang, Phys. Rev. Lett. 7, 46 (1961).

[3] F. Bloch, Phys. Rev. B 2, 109 (1970).

[4] M. Büttiker, Y. Imry, and R. Landauer, Phys. Lett. A 96, 365 (1983).

[5] L. P. Lévy, G. Dolan, J. Dunsmuir, and H. Bouchiat, Phys. Rev. Lett. 64, 2074 (1990).

[6] V. Chandrasekhar et al., Phys. Rev. Lett. 67, 3578 (1991).

[7] D. Mailly, C. Chapelier, and A. Benoit, Phys. Rev. Lett. 70, 2020 (1993).

[8] A. Fuhrer et al., Nature (London) 413, 822 (2001).

[9] J. M. García et al., Appl. Phys. Lett. 71, 2014 (1997).

[10] D. Granados and J. M. García, Appl. Phys. Lett. 82, 2401 (2003).

[11] M. Bayer et al., Phys. Rev. Lett. 90, 186801 (2003).

[12] R. J. Warburton et al., Nature (London) 405, 926 (2000).

[13] A. Lorke et al., Phys. Rev. Lett. 84, 2223 (2000).

[14] P. Offermans et al., Appl. Phys. Lett. 87, 131902 (2005).

[15] D. Granados and J. M. García, Appl. Phys. Lett. 86, 071918 (2005).

[16] G. L. Snider, I. H. Tan, and E. L. Hu, J. Appl. Phys. 68, 2849 (1990).

[17] K. Shiraishi, Appl. Phys. Lett. 60, 1363 (1992).

[18] M. R. Schaapman et al., Appl. Phys. Lett. 81, 1041 (2002).

[19] See EPAPS Document No. E-PRLTAO-99-027741 for more experimental data and a more extensive description of the theoretical calculations. A detailed paper on the theoretical analysis has been submitted for publication elsewhere. For more information on EPAPS, see http:// www.aip.org/pubservs/epaps.html.

[20] J. P. Eisenstein et al., Phys. Rev. Lett. 55, 875 (1985).

[21] M.P. Schwarz et al., Phys. Rev. B 65, 245315 (2002).

[22] M. Zhu et al., Phys. Rev. B 67, 155329 (2003).

[23] L. Landau, Z. Phys. 64, 629 (1930).

[24] M. Grundmann, O. Stier, and D. Bimberg, Phys. Rev. B 52, 11969 (1995).

[25] C. G. Van de Walle, Phys. Rev. B 39, 1871 (1989).

[26] G. L. Bir and G. E. Pikus, Symmetry and Strain-Induced Effects in Semiconductors (John Wiley \& Sons, New York, 1974).

[27] P. Offermans et al., Physica (Amsterdam) 26E, 236 (2005).

[28] V. M. Fomin et al. (to be published). 